Enchi Liu, PhD

Mark E. Schmidt, MD

Richard Margolin, MD

Reisa Sperling, MD,

MMSc

Robert Koeppe, PhD

Neale S. Mason, PhD

William E. Klunk, MD,

$\mathrm{PhD}$

Chester A. Mathis, PhD

Stephen Salloway, MD, MS

Nick C. Fox, MD, FRCP

Derek L. Hill, PhD

Andrea S. Les, PhD

Peter Collins, BA

Keith M. Gregg, PhD

Jianing Di, PhD

Yuan Lu, MS

I. Cristina Tudor, $\mathrm{PhD}$

Bradley T. Wyman, PhD

Kevin Booth, MD, DVM

Stephanie Broome, $\mathrm{PhD}$

Eric Yuen, MD

Michael Grundman, MD, $\mathrm{MPH}$

H. Robert Brashear, MD

For the Bapineuzumab

301 and 302 Clinical

Trial Investigators

Correspondence to

Dr. Liu:

eliu12@its.jnj.com

Supplemental data at Neurology.org

\title{
Amyloid- $\beta{ }^{11} \mathrm{C}-\mathrm{PiB}-\mathrm{PET}$ imaging results from 2 randomized bapineuzumab phase 3 AD trials
}

\section{ABSTRACT}

Objective: To evaluate the effects of bapineuzumab on brain $\beta$-amyloid $(A \beta)$ burden using ${ }^{11} \mathrm{C}$-Pittsburgh compound B $\left({ }^{11} \mathrm{C}-\mathrm{PiB}\right)-\mathrm{PET}$.

Methods: Two phase 3 clinical trials, 1 each in apolipoprotein APOE $\varepsilon 4$ carriers and noncarriers, were conducted in patients with mild to moderate Alzheimer disease dementia. Bapineuzumab, an anti-A $\beta$ monoclonal antibody, or placebo, was administered by IV infusion every 13 weeks for 78 weeks. PET substudies assessed change in brain fibrillar A $\beta$ over 71 weeks using an ${ }^{11} \mathrm{C}$-PiB-PET standardized uptake value ratio (SUVr) global cortical average (GCA) comprising the average SUVr from 5 cortical regions of interest with cerebellar gray matter as the reference region.

Results: A total of 115 carriers and 39 noncarriers were analyzed. The difference $(\delta)$ in mean baseline to 71 week change in ${ }^{11} \mathrm{C}-\mathrm{PiB}-\mathrm{PET}$ GCA between bapineuzumab and placebo was significant in carriers $(0.5 \mathrm{mg} / \mathrm{kg}$ vs placebo $\delta=-0.101 ; p=0.004)$ and in pooled analyses of both carriers and noncarriers $(0.5 \mathrm{mg} / \mathrm{kg}$ vs placebo $\delta=-0.068 ; p=0.027 ; 1.0 \mathrm{mg} / \mathrm{kg}$ vs placebo $\delta=-0.133 ; p=0.028$ ) but not in the noncarrier trial separately. Analyses by individual region of interest and in mild disease yielded findings similar to the main trial results.

Conclusions: The ${ }^{11} \mathrm{C}-\mathrm{PiB}-\mathrm{PET}$ imaging results demonstrated reduction of fibrillar $\mathrm{A} \beta$ accumulation in patients with Alzheimer disease treated with bapineuzumab; however, as no clinical benefit was observed, the findings are consistent with the hypotheses that bapineuzumab may not have been initiated early enough in the disease course, the doses were insufficient, or the most critical $A \beta$ species were inadequately targeted. Neurology ${ }^{\circledR} 2015 ; 85: 692-700$

\section{GLOSSARY}

$\mathbf{A} \boldsymbol{\beta}=\beta$-amyloid $\mathbf{A D}=$ Alzheimer disease; $\mathbf{A R I A}=$ amyloid-related imaging abnormalities; $\mathbf{G C A}=$ global cortical average; MMSE = Mini-Mental State Examination; NINCDS-ADRDA = National Institute of Neurological and Communicative Disorders and Stroke-Alzheimer's Disease and Related Disorders Association; PiB = Pittsburgh compound B; ROI = region of interest; $\mathbf{S U V r}=$ standardized uptake value ratio.

Bapineuzumab, a humanized monoclonal antibody targeting the $\mathrm{N}$-terminus of $\beta$-amyloid $(A \beta)$, was recently evaluated in phase 3 trials for the treatment of mild to moderate Alzheimer disease $(\mathrm{AD})$ dementia. As part of those investigations, brain volumetric MRI, brain amyloid PET imaging, and CSF sampling were performed in biomarker substudies. The primary aim of the substudies was to assess the pharmacologic effects of bapineuzumab on AD CNS biomarkers. The PET substudy used ${ }^{11} \mathrm{C}$-Pittsburgh compound B $\left({ }^{11} \mathrm{C}-\mathrm{PiB}\right)-\mathrm{PET}$ as a measure of brain fibrillar $A \beta .{ }^{1}$ Differences in the incidence of amyloid-related imaging abnormalities (ARIA) and potential efficacy signals had been seen between participants treated with bapineuzumab who were $A P O E \& 4$ carriers and noncarriers in phase 2 studies $^{2-4}$; therefore, separate clinical trials for $A P O E \& 4$ carriers (Study 302) and noncarriers (Study 301) were conducted in phase 3. The primary clinical and biomarker endpoint results of these trials were recently

\footnotetext{
All authors contributed equally to this work.

From Janssen Alzheimer Immunotherapy Research \& Development, LLC (E.L., R.M., P.C., K.M.G., J.D., Y.L., I.C.T., S.B., E.Y., H.R.B.), South San Francisco, CA; Janssen Pharmaceutical (M.E.S.), Beerse, NV; Brigham \& Women’s Hospital (R.S.), Boston, MA; University of Michigan (R.K.), Ann Arbor; University of Pittsburgh (N.S.M., W.E.K., C.A.M.), PA; Butler Hospital (S.S.), Providence, RI; UCL Institute of Neurology (N.C.F.), London, UK; IXICO plc (D.L.H., A.S.L.), London, UK; Pfizer Inc. (B.T.W.), Groton, CT; Pfizer Inc. (K.B.), Collegeville, PA; Global R\&D Partners, LLC (M.G.), San Diego, CA; and University of California (M.G.), San Diego.

Bapineuzumab Study 301 and 302 study investigators are listed on the Neurology ${ }^{\circledR}$ Web site at Neurology.org.

Go to Neurology.org for full disclosures. Funding information and disclosures deemed relevant by the authors, if any, are provided at the end of the article.
} 
reported. ${ }^{5}$ We report additional analyses of the ${ }^{11} \mathrm{C}$-PiB-PET data that evaluate the effects of $A P O E \& 4$ status and disease severity, pooled analyses of the carrier and noncarrier studies, individual regions of interest (ROI), and a sensitivity analysis using the pons as a reference region instead of cerebellar gray matter.

METHODS Patients. Enrollment criteria and randomization scheme for the bapineuzumab PiB-PET substudies were the same as for the main studies described previously. ${ }^{5}$ In brief, eligible patients were aged 50-88 years inclusive, met National Institute of Neurological and Communicative Disorders and Stroke-Alzheimer's Disease and Related Disorders Association (NINCDS-ADRDA) clinical criteria for probable $\mathrm{AD},{ }^{6}$ had a Mini-Mental State Examination (MMSE) ${ }^{7}$ score of 16-26, and had a modified Hachinski Ischemic Score $\leq 4 .{ }^{8}$ Patients were excluded for clinically significant neurologic disease other than $\mathrm{AD}$.

Standard protocol approvals, registrations, and patient consents. The institutional review board for each site approved the study, and each participant (or legally authorized representative) gave written informed consent before enrollment.

In the $A P O E \& 4$ carrier study, 1,121 participants were randomized in a ratio of $3: 2$ bapineuzumab $0.5 \mathrm{mg} / \mathrm{kg}$ placebo. In the noncarrier study, 1,331 participants were randomized in a ratio of $1: 1: 1: 2$ bapineuzumab $0.5 \mathrm{mg} / \mathrm{kg} 1.0 \mathrm{mg} / \mathrm{kg} 2.0 \mathrm{mg} / \mathrm{kg}$ placebo. However, the $2.0 \mathrm{mg} / \mathrm{kg}$ dose was discontinued early in the trial owing to safety events (symptomatic ARIA); participants randomized to that dose were excluded from the PET substudy analysis (figure e-1 on the Neurology ${ }^{(B)}$ Web site at Neurology.org). Randomization stratification factors were current cholinesterase inhibitor or memantine use; baseline MMSE total score (low = 16-21 vs high =22-26); and, in the carrier study, APOE $\varepsilon 4$ copy number (1 vs 2). Patients received study drug as a 1 -hour IV infusion every 13 weeks during the 18 -month study.

The primary objective of the phase 3 studies was to evaluate the efficacy of bapineuzumab administered intravenously compared with placebo by measuring the change from baseline to week 78 in clinical endpoints. An important secondary objective was to evaluate the effect of bapineuzumab on change in standardized uptake value ratio (SUVr) from baseline to week 71 in brain $\mathrm{A} \beta$ burden using ${ }^{11} \mathrm{C}-\mathrm{PiB}-\mathrm{PET}$ in a substudy of approximately $10 \%$ of enrolled participants. Exploratory objectives were to evaluate the effect of bapineuzumab on ${ }^{11} \mathrm{C}-\mathrm{PiB}-\mathrm{PET}$ change in participants with mild and moderate disease separately (defined by baseline MMSE ranges of 22-26 and 16-21, respectively) in pooled analyses using all analyzable patients from both the carrier and noncarrier studies and in individual brain ROIs. Finally, the effect of using the pons as an alternative reference region to the cerebellar gray matter was evaluated in a prespecified analysis.

${ }^{11} \mathrm{C}$-PiB-PET scans were obtained at baseline, week 45 , and week 71. Imaging was conducted at 14 US academic PET centers, almost all having prior experience with ${ }^{11} \mathrm{C}-\mathrm{PiB}-\mathrm{PET}$ imaging in $\mathrm{AD}$. All sites underwent a study-specific site qualification process. Several different PET or PET/CT cameras were used although centers were requested to scan participants with the same camera throughout the study. Cameras had to be capable of emission data collection in 3 dimensions and have an axial field of view sufficient for imaging the entire brain in a single bed position. Centers had to provide Hoffman phantom data of suitable quality. Camera-specific acquisition, emission correction, and reconstruction settings were adopted from the Alzheimer's
Disease Neuroimaging Initiative (http://www.adni-info.org/ Scientists/ADNIStudyProcedures.aspx). Best local practices were used to ensure that participants remained in the field of view with minimal movement during scanning.

${ }^{11} \mathrm{C}-\mathrm{PiB}$ was prepared in accordance with documented manufacturing and control information, and quality was reviewed during the course of the trial. A target ${ }^{11} \mathrm{C}-\mathrm{PiB}$ dose of $555 \mathrm{MBq}$ (15 mCi) (accepted range: $370-610 \mathrm{MBq}[10-16.5 \mathrm{mCi}]$ ) was injected over $\leq 30$ seconds. Emission data were collected as four 5-minute frames from 50 to 70 minutes post-tracer injection ${ }^{1}$; attenuation data were acquired using procedures appropriate to PET-only or PET/CT cameras and were used for image reconstruction by the site. Baseline 3D T1 MRI scans for each participant were obtained for use in PiB-PET analyses.

Reconstructed PET scans and baseline 3D T1 MRI scans for each participant were transferred from acquisition sites for quality review and only images determined to be of suitable quality for quantitative analysis were included.

Image analysis. The second through fourth frames comprising the reconstructed ${ }^{11} \mathrm{C}$-PiB-PET scans for each participant were rigidly registered to the first frame to account for movement between frames and then averaged.

The BrainWeb 3D T1 MRI (http://brainweb.bic.mni.mcgill. ca/brainweb/) $)^{9}$ was nonrigidly registered to the participant's $3 \mathrm{D}$ T1 MRI, ${ }^{10,11}$ and this transformation was used to map the following ROIs to the participant's 3D T1 MRI: the Automated Anatomical Labelling atlas, ${ }^{12}$ an ROI defined for cerebellar gray matter with the inferior $10 \mathrm{~mm}$ truncated to exclude less reliable emission data from the edge of the field of view, and the pons. The participant's 3D T1 MRI was then segmented into gray and white matter tissue masks. ${ }^{13}$ Finally, the participant's 3D T1 MRI was rigidly registered to the PET, and this transformation was used to map all ROIs and tissue masks onto the participant's registered and averaged PET. Final ROIs were defined as the intersection of the ROIs and tissue masks in PET space.

Five cerebral regions known to accumulate substantial fibrillar $\mathrm{A} \beta$ in $\mathrm{AD}$ (anterior cingulate, posterior cingulate/precuneus, frontal, lateral temporal, and parietal cortex) were considered primary for evaluating treatment effects. Individual regional SUVrs were calculated as the ratio of tissue activity for each ROI to the tissue activity of the reference region: cerebellar gray matter for the prespecified analysis in the statistical analysis plan and pons as an additional prespecified analysis in the study imaging charter. The global cortical average (GCA) SUVr was constructed from the unweighted average of the SUVrs from these 5 individual cerebral ROIs. Additional ROIs (mesial temporal cortex, occipital, striatum, and thalamus) were included in separate individual ROI analysis.

Sample size. In the carrier study, acquisition of PiB-PET data for 32 participants in the placebo group and 48 participants in the bapineuzumab group was estimated to provide $90 \%$ power to detect a 0.152 SUVr unit reduction in $A \beta$ burden for the bapineuzumab group compared with placebo at week 71 . In the noncarrier study, 45 participants in the placebo group and 25 participants in each of the 2 bapineuzumab groups were estimated to provide $>90 \%$ power to detect a 0.186 SUVr unit reduction in amyloid burden for a bapineuzumab group compared with placebo at week 71 . The sample size calculations were based on 2 -sided tests with $\alpha$ set at 0.05 . The standard deviations for both studies (0.203) were estimated from a previous bapineuzumab study. ${ }^{3}$

Analysis specifications. ${ }^{11} \mathrm{C}-\mathrm{PiB}-\mathrm{PET}$ GCA analyses were performed on a modified intent-to-treat population and included 
Figure 1 Distribution of ${ }^{11} \mathrm{C}$-Pittsburgh compound B-PET global cortical average at baseline among $A P O E \& 4$ carriers and noncarriers

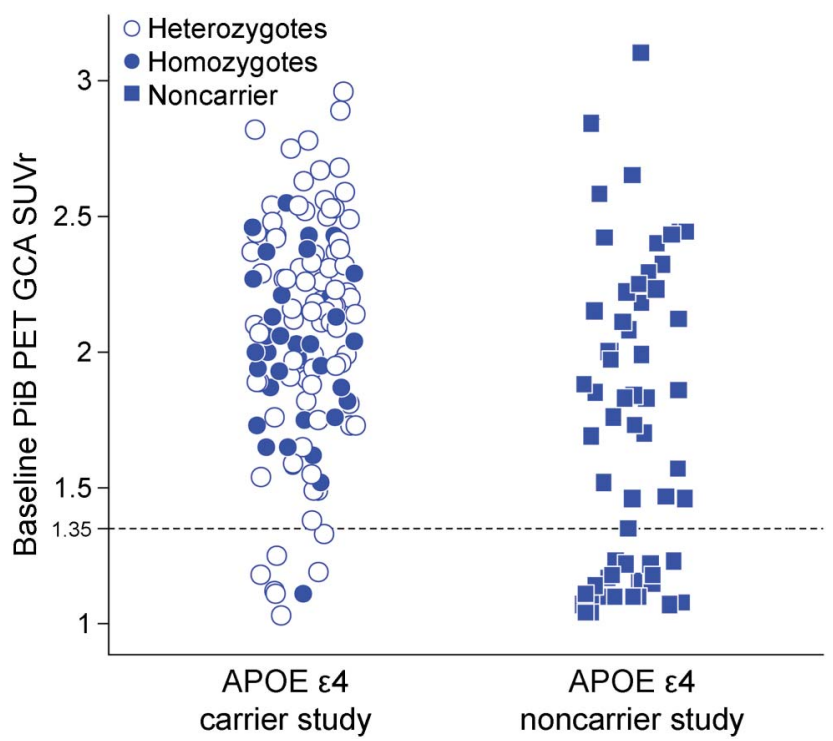

A total of $6.5 \%(8 / 123)$ of APOE $\varepsilon 4$ carriers had baseline scans with global cortical average (GCA) $<1.35$ compared with $36.1 \%(21 / 61)$ of noncarriers. PiB = Pittsburgh compound B; $\mathrm{SUVr}=$ standardized uptake value ratio.

only participants who received at least part of 1 infusion and had a baseline, and at least 1 postbaseline, ${ }^{11} \mathrm{C}-\mathrm{PiB}-\mathrm{PET}$ assessment.

Change-from-baseline analyses were further restricted to participants who also had a baseline GCA $\geq 1.35$ (referred to as the PiB-PET analysis population) to allow for a potential reduction in ${ }^{11} \mathrm{C}-\mathrm{PiB}$ to be observed. ${ }^{11} \mathrm{C}-\mathrm{PiB}$ GCA values $<1.35$ were considered amyloid-negative.

Unless otherwise noted, change from baseline was analyzed using a restricted maximum likelihood-based mixed model for repeated measures. The response variable was the change from baseline to each postbaseline visit, and the primary endpoint was the change from baseline to week 71 . The model included the following fixed effects: treatment (with 2 levels, bapineuzumab and placebo, in carriers; with 3 levels, bapineuzumab $0.5 \mathrm{mg} / \mathrm{kg}$, bapineuzumab $1.0 \mathrm{mg} / \mathrm{kg}$, and placebo, in noncarriers and pooled analyses), visit (scheduled, a categorical factor), treatment-by-visit interaction, baseline GCA (a numerical covariate), baseline-byvisit interaction, baseline MMSE total score stratum (with 2 levels, low $\leq 21$ and high $\geq 22$ ), baseline cholinesterase inhibitor or memantine use stratum (with 2 levels, yes and no), and baseline age (a numerical covariate). APOE $\& 4$ copy number stratum was included in the carrier study (with 2 levels, 1 copy and 2 copies) whereas $A P O E \& 4$ carrier status (carrier or noncarrier) was included in the pooled study analyses. An unstructured variancecovariance structure was used to model the within-subject errors. Estimates are reported as mean \pm SE unless otherwise noted. Reported $p$ values are unadjusted for multiple comparisons; $p$ values $<0.05$ were considered nominally significant.

RESULTS Participant disposition is summarized in figure e-1. In the carrier study, 155 of 1,121 randomized participants were enrolled in the placebo $(\mathrm{n}=$ $60)$ and $0.5 \mathrm{mg} / \mathrm{kg}(\mathrm{n}=95)$ treatment groups of the PET substudy. In the noncarrier study, 84 of 1,331 randomized participants were enrolled in the placebo $(\mathrm{n}=33), 0.5-\mathrm{mg} / \mathrm{kg}(\mathrm{n}=26)$, and $1.0-\mathrm{mg} / \mathrm{kg}$ $(\mathrm{n}=25)$ treatment groups of the PET substudy. The mean (SD) baseline GCA was 2.07 (0.403) for carriers and $1.72(0.548)$ for noncarriers $(p<0.001$, $t$ test). The baseline GCA distributions for carriers and noncarriers with postbaseline PET measures are illustrated in figure 1. Data for $6.5 \%$ of carriers and $36.1 \%$ of noncarriers were excluded from the changefrom-baseline analyses owing to a baseline GCA less than the prespecified threshold for inclusion (1.35), resulting in 115 carrier participants (40 receiving placebo and 75 receiving $0.5 \mathrm{mg} / \mathrm{kg}$ ) and 39 noncarrier participants (15 receiving placebo, 12 receiving $0.5 \mathrm{mg} / \mathrm{kg}, 12$ receiving $1.0 \mathrm{mg} / \mathrm{kg}$ ) in the PiB-PET analysis population. The mean (SD) baseline GCAs were similar for carriers $(2.14 \pm$ $0.333)$ and noncarriers $(2.05 \pm 0.400)$ in the PiBPET analysis population $(p=0.18)$. The baseline demographics, clinical characteristics, and GCAs of the PiB-PET analysis population were similar between the treatment and placebo groups (table 1).

The key PET results for each study, for both studies combined, and for the mild and moderate $\mathrm{AD}$ subgroups, derived using the PiB-PET analysis population, are shown in figure 2 (figures e- 2 and e-3 show individual participant data). Among carriers (figure $2 \mathrm{~A}$ ), a baseline to 71 week increase in GCA was observed in the placebo group (mean \pm SE $0.102 \pm$ 0.026 ) but not in the bapineuzumab group (mean \pm SE $0.001 \pm 0.021$ ), resulting in a significant treatment difference $(\delta)$ in the baseline to week 71 change in mean GCA between groups $(\delta=-0.101 ; p=$ 0.004). The baseline to week 71 increase (sample mean $\pm \mathrm{SE}$ ) in GCA among placebo carriers was similar among heterozygote $(0.13 \pm 0.034)$ and homozygote APOE $\varepsilon 4$ carriers $(0.08 \pm 0.038)$. In noncarriers, a modest baseline to week 71 decrease in mean GCA was observed in the placebo group $(-0.046 \pm 0.0443)$, with no statistically significant treatment differences for either the $0.5-\mathrm{mg} / \mathrm{kg}(\delta=0.085, p=0.193)$ or the $1.0-\mathrm{mg} / \mathrm{kg}$ groups $(\delta=-0.048, p=0.466)$ compared with placebo (figure $2 \mathrm{~B}$ ). In the pooled study analysis that included participants from both studies (figure 2C), the baseline to week $71{ }^{11} \mathrm{C}-\mathrm{PiB}-\mathrm{PET}$ changes for placebo, $0.5 \mathrm{mg} / \mathrm{kg}$, and $1.0 \mathrm{mg} / \mathrm{kg}$, respectively, were $0.072 \pm 0.023,0.004 \pm 0.019$, and $-0.061 \pm 0.055$, with treatment differences compared with placebo observed for both the $0.5-\mathrm{mg} / \mathrm{kg}$ $(\delta=-0.068 ; p=0.027)$ and $1.0-\mathrm{mg} / \mathrm{kg}(\delta=$ $-0.133 ; p=0.028)$ doses. The treatment differences observed in the carrier study and in the pooled analysis were most pronounced in the mild subgroup (figure 2, $\mathrm{D}$ and $\mathrm{F})$. No significant differences were observed in the moderate subgroup, either in the individual study or pooled study analyses.

The treatment-related difference in mean GCA observed from baseline to week 71 among carriers 
Table $1 \quad{ }^{11} \mathrm{C}-\mathrm{PiB}-\mathrm{PET}$ analysis population ( $\geq 1.35 \mathrm{SUVr}$ ): Demographic and baseline characteristics

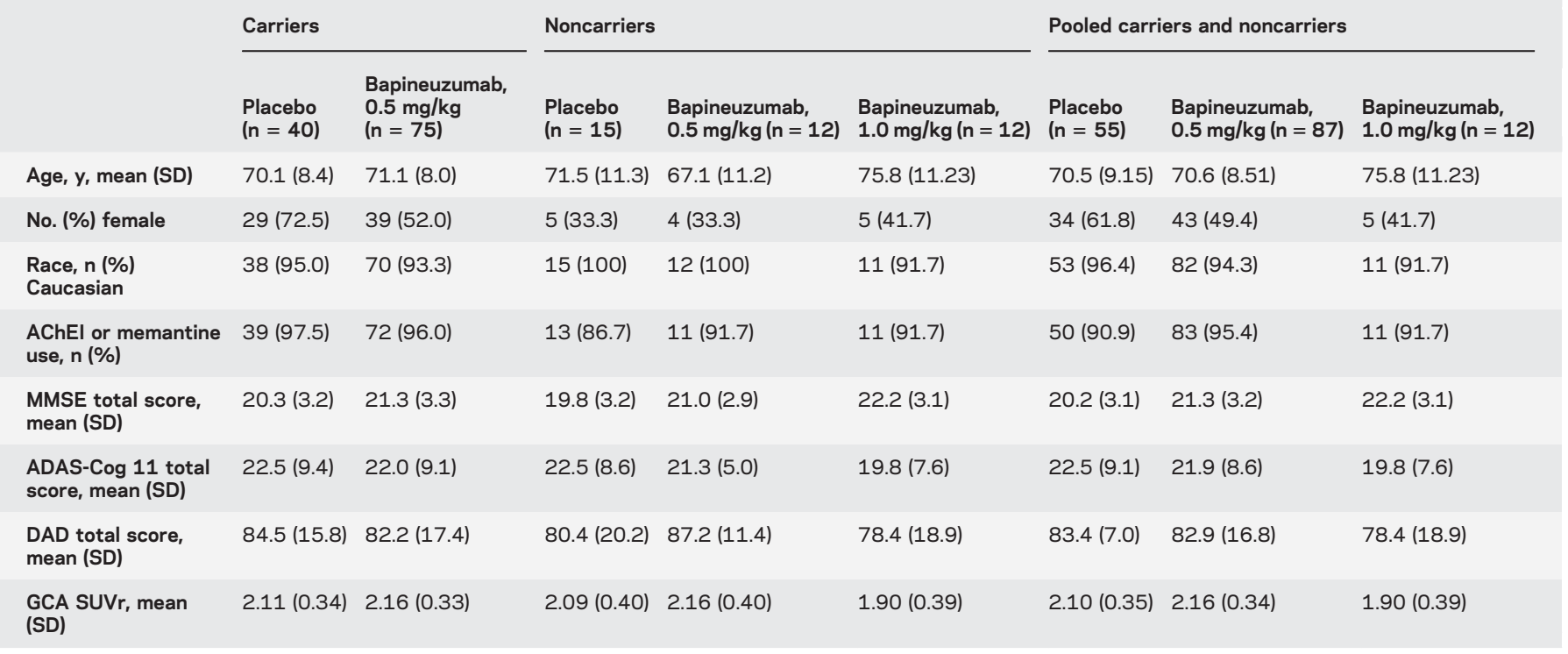

Abbreviations: $\mathrm{AChEI}=$ acetylcholinesterase inhibitor; ADAS-Cog $=$ Alzheimer's Disease Assessment Scale-Cognitive component; $\mathrm{DAD}=\mathrm{Disability}$ Assessment for Dementia; GCA SUVr = global cortical average standardized uptake value ratio; MMSE = Mini-Mental State Examination; PiB = Pittsburgh compound B; SUVr = standardized uptake value ratio.

was consistent with the 5 individual ROI analyses (table 2). Treatment-related differences in the change from baseline to week 71 regional SUVrs were observed in the anterior cingulate, posterior cingulate/precuneus, and lateral temporal cortices, with trends observed in the frontal and parietal cortices. Treatment-related differences in the change from baseline to week 71 SUVrs were also observed in the mesial temporal and occipital cortices and subcortical regions of the thalamus and striatum. The lack of treatment-related differences in GCA change over 71 weeks observed in the noncarrier study was also seen in the individual region analysis, except for the subcortical regions at the $1.0 \mathrm{mg} / \mathrm{kg}$ dose, in which treatment-related differences in the change from baseline to week 71 SUVrs were observed (table 2).

Pooling data from the carrier and noncarrier studies, there was no evidence of ${ }^{11} \mathrm{C}-\mathrm{PiB}-\mathrm{PET}$ accumulation among $A \beta$-negative participants (baseline GCA $<1.35)$ at week 71 , among either the placebo or treated groups (sample mean GCA change from baseline $=0.00 \pm 0.011$ for placebo, $0.01 \pm 0.011$ for treated participants).

A sensitivity analysis of the change in the mean GCA was also performed for each study using the pons as a reference region (figure 3). For carriers, there was a small increase in mean GCA for the placebo group $(0.021 \pm 0.0130)$, and a small decrease for the $0.5 \mathrm{mg} / \mathrm{kg}$ group $(-0.023 \pm 0.0102)$, resulting in a significant treatment-related difference in the baseline to week 71 change in GCA ( $\delta=-0.044$, $p=0.011$ ). For noncarriers, there were no significant treatment-related differences in the baseline to week
71 change in ${ }^{11} \mathrm{C}$-PiB-PET GCA for the $0.5-\mathrm{mg} / \mathrm{kg}$ $(\delta=-0.041, p=0.387)$ or $1.0-\mathrm{mg} / \mathrm{kg}$ groups $(\delta=-0.085, p=0.083)$; however, in this analysis there was no change in mean GCA SUVr for the placebo group at week $71(0.00 \pm 0.0320)$, in contrast to the modest decrease observed using the cerebellar reference region. Additionally, a modest decrease was observed in the baseline to week 71 change in GCA in the $0.5 \mathrm{mg} / \mathrm{kg}$ group.

DISCUSSION The totality of the data from the 2 phase 3 PET bapineuzumab substudies suggests that IV treatment with bapineuzumab reduced fibrillar $A \beta$ accumulation relative to placebo over 71 week in mild to moderate AD dementia. This reduction was most clearly evident in the carrier study and the pooled analyses (which included both carriers and noncarriers) for both the $0.5-$ and $1.0-\mathrm{mg} / \mathrm{kg}$ doses. The observation that the treatment differences seen in the carrier and pooled analyses seem to have been driven primarily by participants with mild disease raises the possibility that bapineuzumab immunotherapy could have a greater effect on amyloid accumulation in patients treated earlier in the disease course. These results, together with CSF analyses ${ }^{4}$ performed in patients treated with bapineuzumab, support the hypothesis that bapineuzumab can enter the brain and achieve exposures sufficient to engage its target, thereby altering fibrillar $A \beta$ accumulation.

The prevalence of significant $A \beta$ burden at baseline and subsequent change in GCA over the course of the study was lower for $A P O E \& 4$ noncarriers than carriers. The smaller than expected analyzable sample 

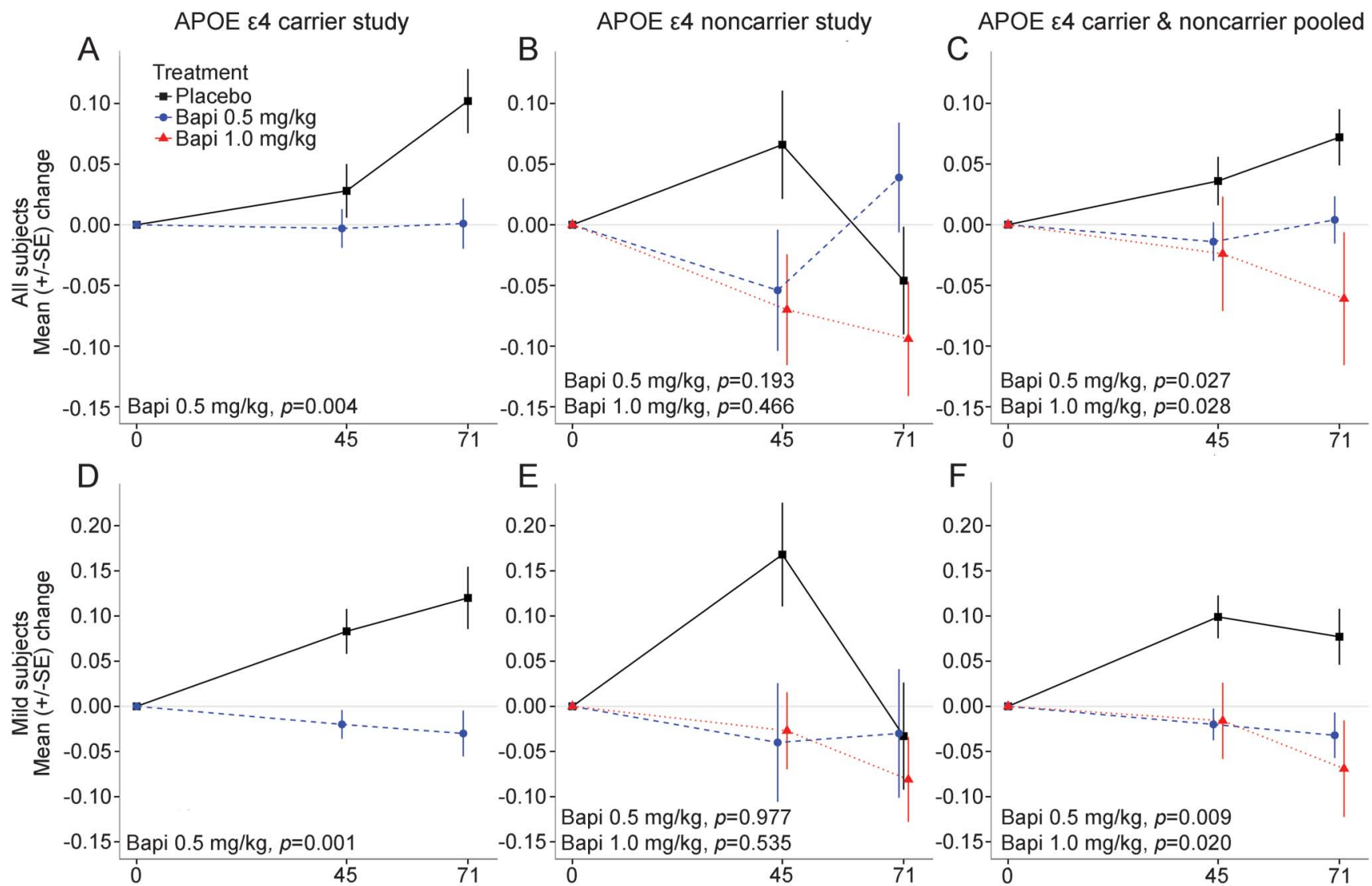

$0.15-$ Bapi $1.0 \mathrm{mg} / \mathrm{kg}, p=0.028$
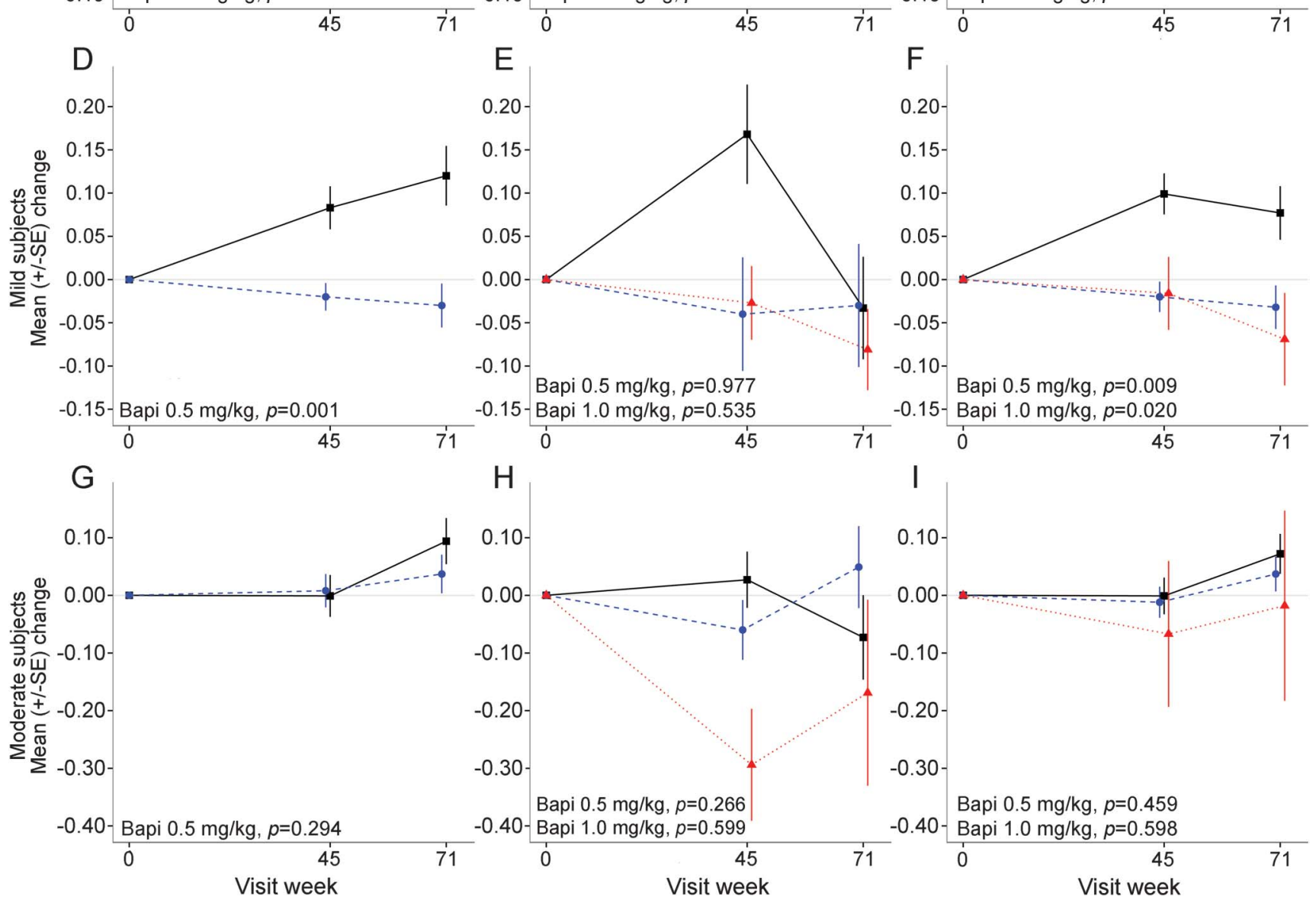

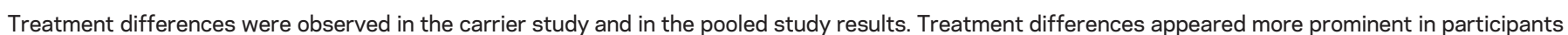

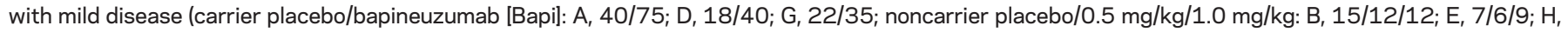
8/6/3; pooled placebo/0.5 mg/kg/1.0 mg/kg: C, 55/87/12; F, 25/46/9; I, 30/41/3).

and lack of ${ }^{11} \mathrm{C}-\mathrm{PiB}$ accumulation over 71 weeks among noncarriers reduced the power of detecting treatment differences in amyloid burden reduction in the noncarrier study. The frequency of amyloidnegative scans in noncarriers $(36 \%)$ in our study is similar to rates observed in phase 2 trials of florbetaben ${ }^{14}$ and florbetapir ${ }^{15}$ and phase 3 studies of solanezumab. ${ }^{16}$ The GCA in amyloid-negative participants did not change significantly over the course of the study. Although all participants met NINCDSADRDA criteria for probable $\mathrm{AD}$, the absence of significant $A \beta$ burden in some participants entering the trial suggests that other disease processes might account for their cognitive impairment. Among placebo participants with baseline GCA $\geq 1.35$, carriers showed an increase in ${ }^{11} \mathrm{C}-\mathrm{PiB}$ signal over the course of the study, whereas noncarriers did not. Grimmer et al. ${ }^{17}$ also found differences by $A P O E \& 4$ genotype in the progression of the cortical ${ }^{11} \mathrm{C}$-PiB-PET signal in participants with AD over a 2-year interval. This finding may suggest that $A P O E$ \&4 has a sustained influence on $A \beta$ 
Table 2 Model-estimated effect of bapineuzumab on individual region of interest SUVrs

\begin{tabular}{|c|c|c|c|c|c|c|}
\hline Region & \multicolumn{2}{|l|}{ Carriers } & \multicolumn{2}{|c|}{ Noncarriers, $0.5 \mathrm{mg} / \mathrm{kg}$ vs placebo } & \multicolumn{2}{|c|}{ Noncarriers, $1.0 \mathrm{mg} / \mathrm{kg}$ vs placebo } \\
\hline GCA & -0.101 & $0.004^{a}$ & 0.085 & 0.193 & -0.048 & 0.466 \\
\hline Anterior cingulate & -0.162 & $<0.001^{a}$ & 0.129 & 0.138 & -0.101 & 0.256 \\
\hline Posterior cingulate/precuneus & -0.090 & $0.021^{a}$ & 0.110 & 0.171 & -0.048 & 0.560 \\
\hline Frontal cortex & -0.096 & 0.099 & 0.057 & 0.455 & -0.088 & 0.265 \\
\hline Parietal cortex & -0.066 & 0.061 & 0.018 & 0.802 & -0.005 & 0.942 \\
\hline Mesial temporal cortex & -0.075 & $<0.001^{a}$ & 0.044 & 0.230 & -0.038 & 0.315 \\
\hline Occipital cortex & -0.055 & $0.027^{a}$ & 0.080 & 0.124 & -0.009 & 0.865 \\
\hline Thalamus & -0.118 & $<0.001^{a}$ & -0.094 & 0.075 & -0.165 & $0.004^{a}$ \\
\hline
\end{tabular}

Abbreviations: GCA = global cortical average; $\mathrm{SUVr}=$ standardized uptake value ratio.

The treatment difference refers to the estimated difference in the mean baseline to week 71 changes in ${ }^{11} \mathrm{C}$-Pittsburgh compound B-PET global cortical average between the treatment groups.

a Significant.

accumulation and not just on the timing of its initiation and could also have implications regarding the use of amyloid PET in detecting treatment effects targeting $A \beta$ deposition.

Treatment of APOE \&4 carriers was associated with significant differences in the change from baseline GCA over 71 weeks compared with placebo whether cerebellar gray or pons was used as the reference. In carriers, treatment-related decreases in $\mathrm{A} \beta$ burden were also observed in most brain regions when analyzed individually. Treatment of noncarriers with bapineuzumab did not show a significant treatment difference in GCA change to week 71 regardless of whether the cerebellar gray or pons was used as the reference region. The variable pattern of GCA change over time observed with the use of different reference regions (figures $2, \mathrm{~A}$ and $\mathrm{B}$, and 3 ) may indicate that the PiB-PET signal changed in one or both reference regions during the trial. Increased $A \beta$ deposition in placebo or decreased deposition in treated participants within a reference region would lead to an underestimation of the treatment effect. The cause of the pattern of change in the placebo noncarriers (figure 2B) is not completely clear, but

Figure 3 Pons-based standardized uptake value ratio: model-estimated change from baseline to week 71
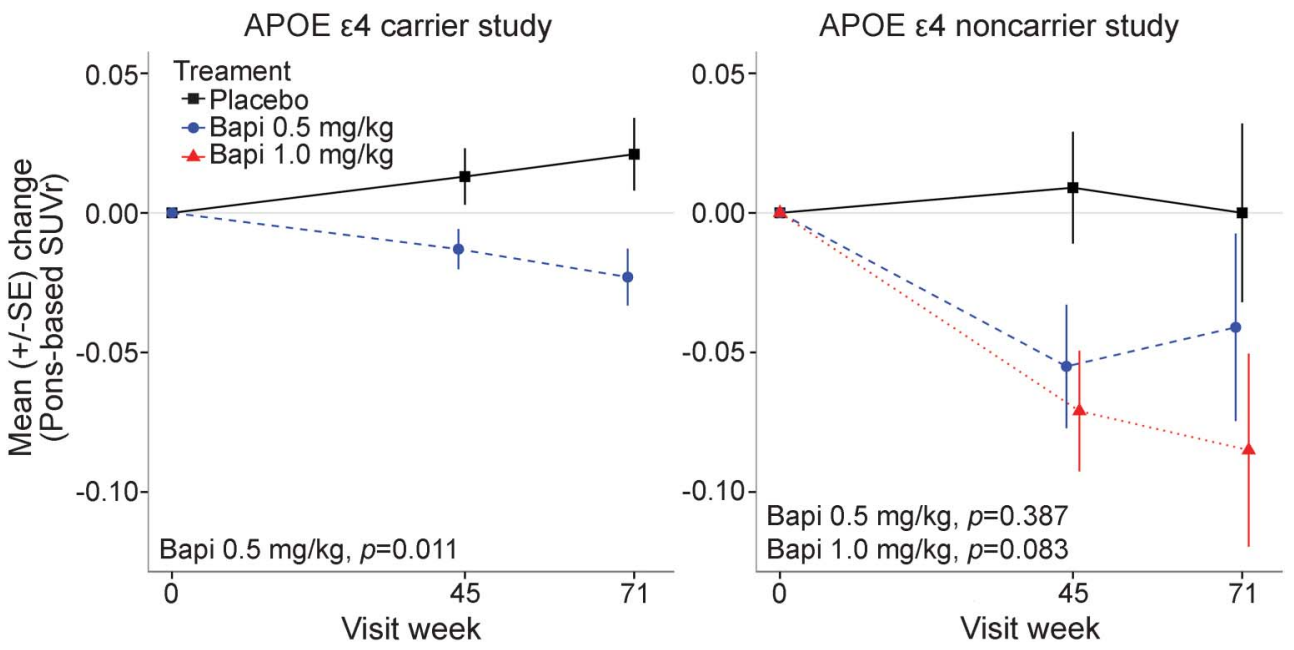

Similar to the results using the cerebellum as a reference region, a treatment difference in carriers was also observed when the pons was used as the reference region (carrier placebo/bapineuzumab [Bapi]: 40/75; noncarrier placebo/0.5 mg/kg/1.0 $\mathrm{mg} / \mathrm{kg}:$ 15/12/12). SUVr = standardized uptake value ratio. 
may also be influenced by one or a combination of factors including the small sample size and impact of technical variability that may have been by introduced by the use of multiple types of scanners in the study and noise from the reference region used in the analysis. ${ }^{18}$

Several studies evaluating AD therapies have incorporated amyloid PET imaging as an outcome measure. In the phenserine trial ${ }^{19}$ and in the solanezumab trials, ${ }^{16}$ no impact of therapy on brain amyloid by PET was observed. In trials with other antiamyloid therapies, ${ }^{3,5,20}$ small to modest treatment reductions on brain amyloid by PET were observed. However, this was not accompanied by clinical benefit ${ }^{5}$ or not reported. ${ }^{20}$ Thus, a question remains as to whether the mechanism of amyloid removal by $\mathrm{AD}$ drugs has really been tested. ${ }^{18-20}$

\section{Comment: \\ Yet another "disconnect" between amyloid and Alzheimer disease?}

Amyloid plaque pathology is a defining characteristic of Alzheimer disease $(\mathrm{AD})$, and the amyloid cascade hypothesis remains a central focus of $\mathrm{AD}$ research. Yet at least a dozen treatment trials have achieved target engagement by reducing fibrillar amyloid or its production, but have shown no clinical benefit. The article by Liu et al. ${ }^{1}$ shows that the monoclonal anti-A $\beta$ antibody bapineuzumab diminished binding of ${ }^{11} \mathrm{C}-\mathrm{Pitts} b u r g h$ compound $\mathrm{B}(\mathrm{PiB})$ in mild to moderate $\mathrm{AD}$ dementia, most notably in persons with $A P O E \& 4$.

The phase III bapineuzumab trials, however, failed to show treatment-related attenuation of cognitive or functional decline. The article by Liu et al. does not discuss that disconnect, perhaps because it is now all too familiar. The rationale usually offered for it is that the intervention was offered too late in the AD pathogenetic cascade.

Speculation is growing, however, regarding another explanation: that fibrillar amyloid (the target of $\mathrm{PiB}$ ) does not cause the symptoms of AD dementia. Newer trials are testing whether anti-amyloid treatments can attenuate the progress of preclinical $\mathrm{AD}$, either in cognitively normal elders with evidence of cerebral amyloidosis $^{2}$ or in those who harbor a pathogenetic PSEN1 mutation. ${ }^{3}$ Contemplation of the eventual results of these trials raises a serious question: just what would constitute compelling evidence that anti-amyloid treatments cannot stop the evolution of the AD pathogenic cascade? Indeed, a null result from the PSEN1 trial would place the severest strain on the entire amyloid cascade hypothesis. The pharmaceutical industry has by now invested several billion dollars in anti-amyloid therapeutics. Karl Popper taught us that scientific discovery requires falsifiable hypotheses. Should we not be asking what could convince us that the anti-amyloid treatment emperor may have no clothes? ${ }^{4}$

1. Liu E, Schmidt ME, Margolin R, et al. Amyloid- $\beta{ }^{11} \mathrm{C}-\mathrm{PiB}-\mathrm{PET}$ imaging results from 2 randomized bapineuzumab phase 3 AD trials. Neurology 2015;85:692-700.

2. Sperling RA, Rentz DM, Johnson KA, et al. The A4 study: stopping AD before symptoms begin? Sci Transl Med 2014;6:228-230.

3. Reiman EM, Langbaum JB, Fleisher AS, et al. Alzheimer's Prevention Initiative: a plan to accelerate the evaluation of presymptomatic treatments. J Alzheimers Dis 2011;26(suppl 3):321-329.

4. The Emporer's New Clothes. Available at: www.andersen.sdu.dk/vaerk/hersholt/ TheEmperorsNewClothes_e.html. Accessed April 17, 2015.

John C.S. Breitner, MD, MPH

From McGill University Faculty of Medicine, Montreal, Canada.

Study funding: No targeted funding reported.

Disclosure: Dr. Breitner has no relevant financial or other competing interests. Within the past 3 years he has been a paid speaker for Lilly, and has consulted to Lundbeck and CereSpir Pharmaceuticals, but on topics unrelated to the content of this article. Go to Neurology.org for full disclosures.
The inability to include higher dose levels (limited by symptomatic ARIA), especially in the carriers, prevented evaluating the potential impact of greater amyloid removal on clinical outcome. Participant enrollment based on clinical criteria manifested in frequent amyloid negatives, especially in the noncarriers, resulting in the reduction of the number of analyzable scans. Although the reported analyses were prespecified, there was no statistical correction for multiple comparisons. Additionally, the pooled study results must be interpreted cautiously, since the $1.0 \mathrm{mg} / \mathrm{kg}$ group consisted only of noncarriers whereas the placebo group comprised both carriers and noncarriers.

In spite of the evidence of target engagement by bapineuzumab, no clinical benefit was evident in the phase 3 trials. ${ }^{5}$ If $\mathrm{A} \beta$ plays a role in the development of $\mathrm{AD}$, these findings raise questions about whether bapineuzumab was initiated early enough to affect the disease course, the exposure was sufficient, or the most pathologic $A \beta$ species were targeted.

\section{AUTHOR CONTRIBUTIONS}

E. Liu: study concept and design, acquisition of data, analysis and interpretation of data, drafting/revising manuscript for content, study supervision and coordination. M. Schmidt: study concept and design, acquisition of data, analysis and interpretation of data, drafting/revising manuscript for content, study supervision and coordination. R. Margolin: study concept and design, acquisition of data, analysis and interpretation of data, drafting/revising manuscript for content, study supervision and coordination. R. Sperling: study concept and design, analysis and interpretation of data, drafting/revising manuscript for content, study supervision and coordination. R. Koeppe: study concept and design, analysis and interpretation of data, drafting/revising manuscript for content, study supervision and coordination. N.S. Mason: study concept and design, analysis and interpretation of data, drafting/revising manuscript for content. W.E. Klunk: study concept and design, analysis and interpretation of data, drafting/revising manuscript for content. C.A. Mathis: study concept and design, analysis and interpretation of data, drafting/revising manuscript for content. S. Salloway: analysis and interpretation of data, drafting/revising manuscript for content, study supervision and coordination. N.C. Fox: analysis and interpretation of data, drafting/revising manuscript for content, study supervision and coordination. D.L. Hill: acquisition of data, analysis and interpretation of data, drafting/revising manuscript for content, study supervision and coordination. A.S. Les: acquisition of data, analysis and interpretation of data, drafting/revising manuscript for content, study supervision and coordination. P. Collins: study concept and design, acquisition of data. K.M. Gregg: analysis and interpretation of data, statistical analysis, drafting/revising manuscript for content. J. Di: analysis and interpretation of data, statistical analysis, drafting/ revising manuscript for content. Y. Lu: analysis and interpretation of data, statistical analysis. I.C. Tudor: study concept and design, analysis and interpretation of data, statistical analysis, drafting/revising manuscript for content. B.T. Wyman: study concept and design, analysis and interpretation of data, drafting/revising manuscript for content. K. Booth: study concept and design, drafting/revising manuscript for content. S. Broome: study concept and design, drafting/revising manuscript for content. E. Yuen: study concept and design, acquisition of data, analysis and interpretation of data, drafting/revising manuscript for content, study supervision and coordination. M. Grundman: study concept and design, acquisition of data, analysis and interpretation of data, drafting/revising manuscript for content, study supervision and 
coordination. H.R. Brashear: study concept and design, acquisition of data, analysis and interpretation of data, drafting/revising manuscript for content, study supervision and coordination.

\section{ACKNOWLEDGMENT}

Some of the software used in the PET SUVr analysis was developed by Sebastian Ourselin, Mark Modat, and Manuel Cardoso at UCL Institute of Neurology and used by IXICO PLC under license from UCL Business, PLC. The authors thank Julia Lull for statistical support and David Lane, Phase Five Communications Inc., for editorial support.

\section{STUDY FUNDING}

Supported by Janssen Alzheimer Immunotherapy and Pfizer, Bapineuzumab 301 and 302 ClinicalTrials.gov numbers NCT00575055 and NCT00574132, EudraCT number 2009-012748-17. Editorial support was funded by Janssen Alzheimer Immunotherapy.

\section{DISCLOSURE}

E. Liu is or was an employee of Janssen Alzheimer Immunotherapy. M. Schmidt is an employee of Janssen Pharmaceutica. R. Margolin is or was an employee of Janssen Alzheimer Immunotherapy. R. Sperling has served as a consultant for Roche, Janssen, Lundbeck, Genentech, and Isis. She has received research support from Eli Lilly and Company and Janssen. R. Koeppe was a consultant to Johnson \& Johnson, Avid, and Merck at the time the manuscript was developed. N. Mason has provided consultancy for Janssen Alzheimer Immunotherapy for the PiB portions of the 301/302 studies related to this manuscript. Dr. Mason has consulted for other pharmaceutical companies, including Elan and Johnson \& Johnson. W. Klunk receives royalty payments from GE Healthcare for licensed PiB-PET technology, has served as a consultant to GE Healthcare and Janssen AI, and has received several grants from the NIH (AG025204, AG025516, AG028526, AG014449, AG005133, and AG012138). C. Mathis receives royalty payments from GE Healthcare for licensed PiB-PET technology. S. Salloway is a consultant to Janssen Alzheimer Immunotherapy, Avid/Lilly, GE Healthcare, AstraZeneca, Biogen, Roche, iPierian, Novartis, Merck, and Piramal, all involving under \$5,000. Dr. Salloway's hospital receives research support for the conduct of clinical trials sponsored by Jansen Alzheimer Immunotherapy, Lilly, Merck, Roche, Functional Neuromodulation, Biogen, Genentech, Avid, and GE Healthcare. N. Fox has provided consultancy to Janssen Alzheimer Immunotherapy and UCL received payment for MR image analysis. His research group has also received payment for consultancy or for conducting studies from AVID, Bristol-Myers Squibb, Elan, Eisai, Lilly, GE Healthcare, IXICO, Johnson \& Johnson, Lundbeck, Novartis, Pfizer, Sanofi, and Wyeth. Dr. Fox receives no personal compensation for the aforementioned activities. D. Hill is an employee of IXICO plc. Dr. Hill holds stock and share options in IXICO plc. Through IXICO, he has provided services to Pfizer, Janssen Alzheimer Immunotherapy, Bristol-Myers Squibb, Lilly, Teva, Roche, MedImmune, GlaxoSmithKline, Kyowa Hakko Kirin, Green Valley, Eisai, VirtualScopics, and Enkam. Dr. Hill holds non-studyrelated patents in image data management and image analysis. Also through IXICO, he has received research grant support from the European grant sources Innovative Medicines Initiative and FP7, also including in the UK the Technology Strategy Board and the Medical Research Council. A. Les is an employee of IXICO plc. P. Collins is or was an employee of Janssen Alzheimer Immunotherapy. K. Gregg is or was an employee of Janssen Alzheimer Immunotherapy. J. Di is or was an employee of Janssen Alzheimer Immunotherapy. Y. Lu reports no disclosures relevant to the manuscript. C. Tudor is or was an employee of Janssen Alzheimer Immunotherapy. B. Wyman is an employee of Pfizer. K. Booth is an employee of Pfizer. S. Broome is or was an employee of Janssen Alzheimer Immunotherapy. E. Yuen is or was an employee of Janssen Alzheimer Immunotherapy. M. Grundman was a paid consultant to Janssen Alzheimer Immunotherapy in the development of this manuscript and has consulted for other pharmaceutical companies, including Adamas, Acumen, ALSP, AstraZeneca, Avid, Biogen Idec, Bristol-Myers Squibb, Elan,
Johnson \& Johnson, Intellect Neurosciences, iPierian, Lilly, MedImmune, NeuroGenetic Pharmaceuticals, NeuroPhage, Nutricia, Phloronol, Prothena, Probiodrug, QR Pharma, and Teva. H. Brashear is or was an employee of Janssen Alzheimer Immunotherapy. Go to Neurology.org for full disclosures.

Received November 25, 2014. Accepted in final form April 7, 2015.

\section{REFERENCES}

1. Klunk WE, Engler H, Nordberg A, et al. Imaging brain amyloid in Alzheimer's disease with Pittsburgh compound B. Ann Neurol 2004;55:306-319.

2. Salloway S, Sperling R, Gilman S, et al. A phase 2 multiple ascending dose trial of bapineuzumab in mild to moderate Alzheimer disease. Neurology 2009;73: 2061-2070.

3. Rinne JO, Brooks DJ, Rossor MN, et al. ${ }^{11} \mathrm{C}-\mathrm{PiB}$ PET assessment of change in fibrillar amyloid-beta load in patients with Alzheimer's disease treated with bapineuzumab: a phase 2, double-blind, placebocontrolled, ascending-dose study. Lancet Neurol 2010; 9:363-372.

4. Blennow $\mathrm{K}$, Zetterberg $\mathrm{H}$, Rinne JO, et al. Effect of immunotherapy with bapineuzumab on cerebrospinal fluid biomarker levels in patients with mild to moderate Alzheimer disease. Arch Neurol 2012;69:1002-1010.

5. Salloway S, Sperling R, Fox NC, et al. Two phase 3 trials of bapineuzumab in mild-to-moderate Alzheimer's disease. N Engl J Med 2014;370:322-333.

6. McKhann G, Drachman D, Folstein M, Katzman R, Price D, Stadlan EM. Clinical diagnosis of Alzheimer's disease: report of the NINCDS-ADRDA Work Group under the auspices of Department of Health and Human Services Task Force on Alzheimer's Disease. Neurology 1984;34:939-944.

7. Folstein MF, Folstein SE, McHugh PR. "Mini-mental state": a practical method for grading the cognitive state of patients for the clinician. J Psychiatr Res 1975;12: 189-198.

8. Rosen WG, Terry RD, Fuld PA, Katzman R, Peck A. Pathological verification of ischemic score in differentiation of dementias. Ann Neurol 1980;7:486-488.

9. Collins DL, Zijdenbos AP, Kollokian V, et al. Design and construction of a realistic digital brain phantom. IEEE Trans Med Imaging 1998;17:463-468.

10. Rueckert D, Sonoda LI, Hayes C, Hill DL, Leach MO, Hawkes DJ. Nonrigid registration using free-form deformations: application to breast MR images. IEEE Trans Med Imaging 1999;18:712-721.

11. Modat M, Ridgway GR, Taylor ZA, et al. Fast free-form deformation using graphics processing units. Computer Methods Programs Biomed 2010;98:278-284.

12. Tzourio-Mazoyer N, Landeau B, Papathanassiou D, et al. Automated anatomical labeling of activations in SPM using a macroscopic anatomical parcellation of the MNI MRI single-subject brain. Neuroimage 2002;15:273-289.

13. Cardoso MJ, Clarkson MJ, Ridgway GR, et al. LoAd: a locally adaptive cortical segmentation algorithm. Neuroimage 2011;56:1386-1397.

14. Barthel H, Gertz HJ, Dresel S, et al. Cerebral amyloid-beta PET with florbetaben $(18 \mathrm{~F})$ in patients with Alzheimer's disease and healthy controls: a multicentre phase 2 diagnostic study. Lancet Neurol 2011;10:424-435.

15. Johnson KA, Sperling RA, Gidicsin CM, et al. Florbetapir (F18-AV-45) PET to assess amyloid burden in Alzheimer's 
disease dementia, mild cognitive impairment, and normal aging. Alzheimers Dement 2013;9:S72-S83.

16. Doody RS, Thomas RG, Farlow M, et al. Phase 3 trials of solanezumab for mild-to-moderate Alzheimer's disease. N Engl J Med 2014;370:311-321.

17. Grimmer T, Tholen S, Yousefi BH, et al. Progression of cerebral amyloid load is associated with the apolipoprotein E epsilon4 genotype in Alzheimer's disease. Biol Psychiatry 2010;68:879-884.

18. Schmidt ME, Chiao P, Klein G, et al. The influence of biological and technical factors on quantitative analysis of amyloid PET: points to consider and recommendations for controlling variability in longitudinal data. Alzheimers Dement (in press 2015).

19. Kadir A, Andreasen N, Almkvist O, et al. Effect of phenserine treatment on brain functional activity and amyloid in Alzheimer's disease. Ann Neurol 2008;63: 621-631.

20. Ostrowitzki S, Deptula D, Thurfjell L, et al. Mechanism of amyloid removal in patients with Alzheimer disease treated with gantenerumab. Arch Neurol 2012; 69:198-207.

\section{Get Connected. Stay Connected.}

Connect with the American Academy of Neurology's popular social media channels to stay up-todate on the latest news and breakthroughs in neurology, and network with peers and neurology thought leaders. Visit $A A N . c o m / C o n n e c t$.

\section{It's Time to Plan for ICD-10, and the AAN Can Help}

All health care providers are required to transition to ICD-10 on October 1, 2015. Claims for services performed on or after this date with an ICD-9 code will not be processed and payments will be delayed. The AAN provides information and resources to help you make this a smooth transition, and has partnered with Complete Practice Resources to provide you with an affordable online project management tool to help walk you through each phase of the necessary preparation to ensure you're ready. Learn more at $A A N$.com/view/ICD10 and start your transition today!

\section{Call for Submissions: Global Perspectives!}

Section Co-Editors Johan A. Aarli, MD, and Oded Abramsky, MD, PhD, FRCP, encourage submissions to the Global Perspectives section that provides a platform in Neurology for news about scientific findings or academic issues. News may include international research content, spotlights on specific neurologic practice concerns within a country, or important information about international educational or scientific collaborative efforts.

Submissions must be 1,250 words or less with five or less references. A maximum of two figures or two tables (or combination) can be incorporated if necessary. For complete submission requirements, please go to Neurology.org and click on "Information for Authors." The submissions will be reviewed by the editors and may be edited for clarity.

Interested submitters can register and upload manuscripts under the section "Global Perspectives" at http://submit.neurology.org. Please send inquiries to Kathy Pieper, Managing Editor, Neurology; kpieper@neurology.org. 